\title{
Literacia em saúde e práticas para prevenção da COVID-19: um estudo transversal
}

\section{em adultos brasileiros}

\author{
Health literacy and prevention practices for COVID-19: a cross-sectional study in Brazilian adults \\ Alfabetización en salud y prácticas para la prevención de COVID-19: un estudio transversal en
} adultos brasileños

Recebido: 24/11/2021 | Revisado: 02/12/2021 | Aceito: 05/12/2021 | Publicado: 10/12/2021

\author{
Bianca Poggianella Fede \\ ORCID: https://orcid.org/0000-0003-1157-3691 \\ Faculdade de Medicina de Jundiaí, Brasil \\ E-mail: biancapoggianella@gmail.com \\ Letícia Rufino Artuso \\ ORCID: https://orcid.org/0000-0003-0844-6990 \\ Faculdade de Medicina de Jundiaí, Brasil \\ E-mail: leticiartuso@gmail.com \\ Carla Fabiana Tenani \\ ORCID: https://orcid.org/0000-0001-7203-2763 \\ Universidade Estadual de Campinas, Brasil \\ E-mail:carlatenani@hotmail.com \\ Carolina Matteussi Lino \\ ORCID: https://orcid.org/0000-0001-6686-3296 \\ Universidade Estadual de Campinas, Brasil \\ E-mail: carolinamatteussi@gmail.com \\ Marília Jesus Batista \\ ORCID: https://orcid.org/0000-0002-0379-3742 \\ Faculdade de Medicina de Jundiaí, Brasil \\ Universidade Estadual de Campinas, Brasil \\ E-mail: mariliajbatista@yahoo.com.br
}

\begin{abstract}
Resumo
Objetivo: Avaliar a literacia em saúde da população do município de Jundiaí em relação à adoção de medidas práticas de prevenção à doença COVID-19. Metodologia: Estudo transversal realizado com 290 participantes do inquérito soroepidemiológico sobre a COVID-19, realizado no município de Jundiaí/SP, sendo 150 com testes negativos e 140 com testes positivos. Por meio de ligações telefônicas foi aplicado o questionário "Health Literacy Scale" (HLS-14), durante os meses de outubro de 2020 a abril 2021. Foi realizado teste do Qui-Quadrado (p<0,05). Resultados: A maioria da amostra era de 18 a 39 anos 46,6\% ( $n=135)$, mulheres 63,1\% ( $n=183)$, com ensino superior 43,4\% $(n=126)$ e renda de 3 ou mais salários mínimos 49,3\% (n=143). Quanto a rotina, 62,1\% (n=180) relatou não sair ou apenas por necessidade, $83,4 \%(n=242)$ seguiam com família as orientações e $68,3 \%(n=198)$ o isolamento social. Baixa literacia foi encontrada em 48,6\% (n=141) e houve associação com maior idade (p-valor=0,004) e desconhecer o distanciamento social como prevenção para COVID-19 (p-valor=0,047). Conclusão: Os resultados demonstram que a baixa literacia foi associada ao desconhecimento do isolamento social como medida de prevenção, demonstrando a importância do investimento em políticas públicas com enfoque em literacia em saúde a fim de controlar a pandemia. Palavras-chave: Letramento em saúde; SARS-CoV-2; Epidemiologia; Saúde pública.
\end{abstract}

\begin{abstract}
Objective: Was to assess the health literacy of the population in the city of Jundiai in relation to the adoption of practical measures to prevent the disease COVID-19. Methodology: Cross-sectional study with 290 adults of epidemiological survey about COVID-19, being 150 with negative tests and 140 with positive tests. Through phone calls, it was applied the Health Literacy Scale (HLS-14), between October 2020 and April 2021. The Chi-Square test ( $\mathrm{p}<0,05)$ was performed. Results: The majority of the sample was between 18 to 39 years old $(46,6 \%$, $n=135)$, women $63,1 \%(n=183)$, with higher education $43,4 \%(n=126)$ and income of 3 or more minimum waged 49,3\% (n=143). As for the routine, $62,1 \%(n=180)$ didn't go out or just did it when necessary, 83,4\% $(n=242)$ followed with their families preventive measures and $68,3 \%(\mathrm{n}=198)$ followed social distancing orientation. Low Health literacy was found in $48,6 \%(\mathrm{n}=141)$ and it was associated with older age $(\mathrm{p}=0,004)$ and also with not understanding social distance as a preventive measure for COVID-19 ( $\mathrm{p}=0,047)$. Conclusion: The results showed a connection between low health literacy and lack of awareness about social distance being a preventive measure, demonstrating how important is to invest in public policies of health literacy in order to control the pandemic.
\end{abstract}

Keywords: Health literacy; SARS-CoV-2; Epidemiology; Public health. 


\begin{abstract}
Resumen
Objetivo: Avaluar la alfabetización en salud de la población de la ciudad de Jundiaí en relación con la adopción de medidas prácticas para prevenir la enfermedad COVID-19. Metodología: Estudio transversal realizado con 290 participantes de la encuesta seroepidemiológica sobre COVID-19, realizada en la ciudad de Jundiaí / SP, con 150 pruebas negativas y 140 pruebas positivas. Por llamadas telefónicas, se aplicó el cuestionario "Health Literacy Scale" (HLS-14) desde octubre de 2020 hasta abril de 2021. Se realizó la prueba de Chi-Cuadrado (p <0.05). Resultados: La mayoría de la muestra tenía entre 18 y 39 años 46,6\% $(n=135)$, mujeres $63,1 \%(n=183)$, con grado superior $43,4 \%$ $(\mathrm{n}=126)$ y renta de 3 o más salarios mínimos 49,3\% $(\mathrm{n}=143)$. En cuanto a la rutina, el $62,1 \%(\mathrm{n}=180)$ refirió no salir o simplemente por necesidad, el $83,4 \%(\mathrm{n}=242)$ siguió las pautas con la familia y el $68,3 \%(\mathrm{n}=198)$ el aislamiento social. Se encontró baja alfabetización en el 48,6\% $(n=141)$ y hubo una asociación con la edad avanzada (valor $\mathrm{p}=0,004)$ y el desconocimiento del distanciamiento social como prevención para COVID-19 (valor $\mathrm{p}=0,047$ ). Conclusión: Los resultados demuestran que la baja alfabetización se asoció con la falta de conocimiento sobre el aislamiento social como medida preventiva, lo que demuestra la importancia de invertir en políticas públicas con enfoque en la alfabetización en salud para el control de la pandemia.
\end{abstract}

Palabras clave: Alfabetización en salud; SARS-CoV-2; Epidemiología; Salud pública.

\title{
1. Introdução
}

No dia 31 de dezembro de 2020, foi reportado à Organização Mundial de Saúde (OMS) o aumento de casos de uma pneumonia de etiologia desconhecida, detectados na cidade de Wuhan, província de Hubei na China. O vírus causador da doença foi descoberto e denominado SARS-CoV-2, responsável pela doença COVID-19 (Shen et al., 2020). Na segunda quinzena de março desse mesmo ano foi declarado o estado de pandemia (WHO, 2020). A transmissão do SARS-CoV-2 ocorre por meio do contato físico e com as gotículas de saliva (Yesudhas et al., 2021). Dentre as medidas de prevenção da COVID-19 recomendadas pela OMS, destaca-se higienizar as mãos com água e sabão ou solução alcoólica, manter distanciamento de, pelo menos 1 metro entre indivíduos, evitar locais com aglomeração de pessoas e evitar levar as mãos ao rosto (WHO, 2019).

Estudos indicam que a adoção do distanciamento social e mudanças de comportamento, podem significantemente mitigar os impactos do COVID-19, como demonstrado a partir de dados cruzados de telefonia móvel e testagem em massa dos Estados Unidos durante os períodos de reclusão (Badr et al., 2020; Wellenius et al., 2021). Considerando tais evidências, o governo de São Paulo, estado mais populoso do Brasil, decretou o início da quarentena no dia 24 de março de 2020 , visando à diminuição da circulação de pessoas nas ruas na tentativa de conter a circulação do vírus (Governo do Estado de São Paulo, 2020).

Nesse sentido, o processo de implementação de medidas de controle efetivas e compreensíveis é essencial para combater os novos patógenos, interromper transmissões e salvar pacientes, pois dessa forma os hospitais conseguem ter tempo hábil para realocação de recursos humanos e materiais (Zhang et al., 2020).

Assim, segundo o boletim epidemiológico do Ministério da Saúde de abril de 2020, aproximadamente 37\% da população adulta referem à realização simultânea de todas as condutas de prevenção da COVID-19. Além do isolamento e a higienização das mãos, o uso de máscara foi incorporado ao rol das práticas em saúde para prevenção do coronavírus. Conforme orientação, seu uso reduz a transmissão e aquisição de infecções virais, porém, sabe-se que nem toda a população tem adesão de comportamentos preventivos recomendados (Ministério da Saúde, 2019).

Entretanto, o constante posicionamento negacionista do governo federal contra o distanciamento social tem impactado a capacidade de controle da disseminação da doença pelo país, uma vez que a desinformação aumenta as incertezas e diminui a adesão das pessoas em relação as medidas de prevenção. Assim, a disseminação de notícias e informações em relação à doença, verdadeiras ou falsas foi chamada de "infodemia", impacta principalmente na compreensão em adultos com baixa literacia, como demonstrado em estudo de Okan et al, (2020).

Nesse contexto, podemos destacar que a literacia em saúde (LS) é um conjunto de competências cognitivas e sociais, bem como a capacidade dos indivíduos de acessarem, compreenderem e usarem informações de forma que mantenham e 
promovam boa saúde A literacia é um meio de empoderamento da população, pois confere a estes, autonomia e capacidade de usar as informações de saúde de maneira efetiva. Uma baixa literacia em saúde implica em menor adesão às medidas preventivas, mais hospitalizações e uma maior taxa de mortalidade. Ademais, Nutbeam faz três possíveis classificações da literacia em saúde: literacia funcional, comunicativa e crítica (Nutbeam, 2020).

Considerando o atual cenário pandêmico e a importância da literacia em saúde na tomada de decisão em saúde, faz se necessário investigar a associação da literacia em saude com o conhecimento e adesão das medidas de prevenção da Covid-19. Em situações emergenciais em saúde como no caso da pandemia por coronavírus, espera-se que haja compreensão da responsabilidade social por trás da necessidade da conteção da pandemia (Abdel-Latif et al., 2020). Ainda a desinformação, a sobrecarga dos profissionais de saúde e de sistemas hospitalares que tem ocorrido durante a pandemia, foram fatores que evidenciaram a importância da literacia em saúde, tanto como um meio de agir de forma mais racional e estratégica, quanto para incentivar as pessoas a compreenderem mais sobre viver uma vida mais saudável (Košir \& Sørensen, 2020) justifica o presente estudo.

Assim, o objetivo do presente estudo foi analisar a associação da literacia em saúde da população do município de Jundiaí com o conhecimento e adoção de medidas para prevenção da COVID-19.

\section{Metodologia}

\subsection{Questões éticas}

Esse estudo foi realizado após aprovação da Comissão em Ética e Pesquisa sobre o parecer nº 31748920.1.0000.5412.

\subsection{Tipo e local de estudo}

Trata-se de um estudo analítico de corte transversal conduzido com participantes do Inqúerito Epidemiológico de Soroprevalência da COVID-19 do município de Jundiaí, realizado entre maio e julho de 2020, organizado pela Faculdade de Medicina de Jundiaí e Prefeitura do Município de Jundiaí.

O município de Jundiaí, do Estado de São Paulo, Brasil, apresenta território de 431.207 km², e, em 2017, possui uma população estimada de 409.497 habitantes (Fundação Sistema Educacional de Análise de Dados, 2021). De acordo com o Instituto Brasileiro de Geografia e Estatística (IBGE, 2010), o Índice de Desenvolvimento Humano Municipal (IDHM), no ano de 2010, era 0,822, sendo considerado muito alto.

\subsection{Seleção da amostra}

O tamanho da amostra foi definido a partir da variável de desfecho literacia em saúde. Considerando a prevalência da baixa literacia em saúde como $40 \%$ (Batista et al., 2020), erro aceitável de 2\%, efeito de delineamento de 2 e significância de 5\%, o tamanho amostral mínimo estimado foi de 227 participantes. Visando compensar eventuais perdas, foi somado ao tamanho amostral 20\% e, assim, o tamanho amostral mínimo era de 283 indivíduos, sendo este número arredondado para 300.

Para seleção dos indivíduos que participaram do Inquérito Epidemiológico foi associado um número de identificação A partir do tamanho amostral, e o total de casos positivos que foram 404, para selecionar 150 indivíduos, calculou-se o intervalo amostral 2,69, arredondado para 2,0. Para os testes com resultado negativo, 2029 voluntários faziam parte da amostra e, considerando um $n=150$, o intervalo amostral calculado foi de 13,53, sendo este arredondado para 13. Assim, foi realizada o sorteio a partir destes intervalos amostrais.

Prevendo perdas, foram também selecionados substitutos em caso de recusa ou alteração dos dados telefônicos de acordo com o intervalo amostral previamente calculado. 


\subsection{Health Literacy Scale (HLS-14)}

Para a avaliação da literacia em saúde, utilizou-se o HLS-14. Trata-se de uma ferramenta abrangente, que avalia os três níveis de literacia: funcional, comunicativa e crítica. Este contém 14 questões, que variam num total de 14 a 70 pontos, com 5 possíveis respostas dentro da escala de likert, variando de "concordo muito", "concordo", "nem concordo, nem discordo", "discordo" e "discordo muito". As pontuações dos itens são somadas de modo que a pontuação final possibilita indicar o nível de literacia em saúde (Batista et al., 2020). Para a classificação entre alta e baixa literacia foi considerado ponto de referência a mediana dos valores. O score do $H L S$ - 14 foi somado e a variável LS foi dicotomizada a partir da mediana, que foi 47 , sendo classificada com alta para quem teve valor 47 ou mais, e baixa até 46 pontos.

\subsection{Coleta dos dados}

Os dados do inquérito utilizados para compor o banco de dados da atual pesquisa foram: resultado do teste, dados cadastrais (nome, documento de identificação, endereço e telefones para contato), idade, sexo, grau de escolaridade, sintomas, profissão, nível de conhecimento sobre a doença e práticas de prevenção, como isolamento e higienização das mãos.

Durante os meses de outubro de 2020 a abril de 2021, os participantes foram convidados pelas duas pesquisadoras e estudantes de medicina, a partir de contato telefônico, a participar do estudo, esclarecendo o Termo de Consentimento Livre e Esclarecido (TCLE). Confirmando a decisão de participar, o consentimento (verbal) foi gravado e, em seguida, as alunas aplicaram o questionário $H L S$-14, durante a ligação de até 15 minutos.

Para cada participante cuja ligação fora encaminhada para a caixa postal foram feitas mais outras duas tentativas. Quando atingida as três ligações ou havia recusa em responder o questionário, o participante era substituído pela lista de substitutos, igualmente sorteados no banco de dados do inquérito epidemiológico.

Ao final da coleta de dados foram obtidas 290 respostas, sendo 150 para participantes com testes negativos e 140 para participantes com testes positivos.

\subsection{Variáveis do estudo}

As variáveis utilizadas foram: soroprevalência para o SARS-CoV-2 (positivo, ou negativo); literacia em saúde (alta LS [ $\geq 47]$, ou baixa LS $[\leq 46])$.

Variáveis sociodemográficas e econômicas: idade (em anos [18 a 39, 40 a 59, 60 anos ou mais]); sexo (masculino, ou feminino); escolaridade (ensino fundamental, ou ensino médio, ou ensino superior, ou não respondeu), renda (sem renda ou até 1 salário, ou 1 a 3 salários, ou 3 ou mais salários, ou sem dados).

As variáveis de comportamentos preventivos relacionados à rotina (não sei ou sai para mercado, farmácia e urgências, ou sai para trabalhar, ou não alterou a rotina); orientações preventivas (seguiu as orientações de prevenção e família, ou não seguiu as orientações de prevenção); isolamento (seguiu, ou não seguiu); evitar contato com alguém próximo infectado (não evitar contato, ou evitar contato); conhecimento preventivo do distanciamento social (não manter distanciamento, ou manter distanciamento); uso de máscara (não fez uso de máscaras, ou fez uso de máscaras).

Em relação à autopercepção, variáveis sobre o novo coronavírus e o tratamento (sim, ou não, ou não sei); vacina (não, ou sim, ou não sei); se já existe vacina para a COVID-19 (sim, ou não, ou não sei); e sobre o conhecimento (pouco, ou médio, ou muito).

\subsection{Processamento e análise dos dados}

As respostas coletadas foram preenchidas no editor de planilhas do programa Excel@, com correspondência de todas as informações para formulação do banco de dados completo no programa e conversão para o Statistical Package for the 
Social Sciences (SPSS). Foram realizadas análises descritivas das variáveis estudadas, bem como a obtenção de médias, medianas e desvio padrão. Foram realizadas análises bivariadas entre o desfecho e as variáveis independentes. Realizou-se análise de mediação para verificar o efeito da literacia no resultado do teste rápido para COVID-19 com o PROCESS do SPSS.

O valor de significância adotado no estudo foi de $5 \%$.

\section{Resultados}

Ao final da pesquisa foram obtidas 290 respostas e, deste montante $48,2 \%$ ( $n=140$ ) eram positivos e $51,7 \%$ ( $n=150$ ) negativos para COVID-19 (Tabela 1).

Em relação ao perfil dos participantes, 46,6\% ( $\mathrm{n}=135)$ apresentam faixa etária de 18 a 39 anos, 43,4\% (n=126) têm ensino superior completo, 49,3\% (n=143) 3 ou mais salários-mínimos, e 63,1\% (n=183) são do sexo feminino.

Foram acessadas informações sobre os comportamentos preventivos gerais, quanto à rotina: $62,1 \%(n=180)$ não sair ou sair apenas para mercado, farmácia ou urgências. Quando questionados sobre as orientações preventivas: 83,4\% ( $\mathrm{n}=242$ ) seguiram, junto à família, as orientações de prevenção. Sobre o isolamento social, 198 (n=198) relataram seguir. 
Tabela 1. Características da literacia em saúde e fatores associados à saúde em 290 adultos testados para COVID-19 em Jundiaí, SP, Brasil, 2020/2021.

\section{VARIÁVEIS}

\section{SOCIODEMOGRÁFICO E ECONÔMICO}

Idade (em anos)

18 a 39

40 a 59

60 ou mais

Sexo

Feminino

Renda pessoal

Sem renda ou até 1 salário*

1 a 3 salários*

Sem dados

\section{COMPORTAMENTOS PREVENTIVOS}

\section{Rotina}

Não sai ou sai para mercado, farmácia e urgências

\section{Orientações preventivas}

Seguiu as orientações de prevenção e família

Não seguiu as orientações de prevenção

$\begin{array}{cc}242 & 83,4 \% \\ 48 & 16,6 \%\end{array}$

\section{Isolamento}

Seguiu

Não seguiu

AUTOPERCEPÇÃO SOBRE COVID-19

Em relação ao tratamento

Sim

Não

Não sei

$\begin{array}{cc}56 & 19,3 \% \\ 214 & 73,8 \% \\ 20 & 6,9 \%\end{array}$

Vacina

Não

Sim

Não sei

$\begin{array}{cc}238 & 82,1 \% \\ 34 & 11,7 \% \\ 18 & 6,2 \%\end{array}$

Conhecimento

Pouco

Médio

Muito

Fonte: Elaborada pelos autores.*Salário mínimo brasileiro $=\mathrm{R} \$ 998,00$ (Dez/2020) e R $\$ 1.100,00$ (Mar/2021). **LS bivariada sobre o questionário HLS-14 (Suka et al., 2013) e (Batista et al., 2020) 
No questionário também foram obtidas informações sobre a autopercepção sobre COVID-19, quanto ao tratamento foram questionados se existia tratamento, $73,8 \%(\mathrm{n}=214)$ afirmou que não existia tratamento. Ainda nessa categoria, os participantes responderam à pergunta se na época haviam vacinas disponíveis para a COVID-19, a maioria respondeu não existir $(82,1 \%, \mathrm{n}=238)$. Por fim quando tiveram que fazer uma autoavaliação subjetiva sobre o grau de conhecimento sobre a doença, 69,3\% (n=201) se categorizam em nível médio de conhecimento.

A amostra foi classificada em alta literacia, sendo $51,4 \%(n=149)$ do total e 48,6\% ( $n=141)$ possuem baixa literacia. Nesta amostra evidenciou-se que os participantes apresentaram maior prevalência de "eu concordo" nas perguntas 1, 2 e 3 na dimensão funcional. Já para a literacia comunicativa houve maior prevalência de "discordo ou discordo muito" nas questões 6,7, 9 e 10, e para a crítica em todas as questões. Na Tabela 2, estão expostas as distribuições de resposta para as perguntas do $H L S-14$, de acordo com o tipo de literacia funcional, comunicativa e crítica.

Tabela 2. Distribuição da Literacia em saúde, para cada questão, em 288 adultos testados para COVID-19 em Jundiaí, SP, Brazil, 2020/2021.

\begin{tabular}{|c|c|c|c|c|c|}
\hline \multirow{3}{*}{ QUESTÕES HLS-14 } & \multicolumn{5}{|c|}{ Respostas } \\
\hline & Concordo muito & Concordo & $\begin{array}{l}\text { Nem concordo, } \\
\text { nem discordo }\end{array}$ & Discordo & Discordo muito \\
\hline & $\mathbf{n}(\%)$ & n $(\%)$ & n $(\%)$ & $\mathbf{n}(\%)$ & n $(\%)$ \\
\hline \multicolumn{6}{|l|}{ LITERACIA FUNCIONAL } \\
\hline $\begin{array}{l}\text { 1.Eu encontro palavras que não consigo ler } \\
\text { 2.A impressão é muito pequena para mim }\end{array}$ & $29(10 \%)$ & $184(63,4 \%)$ & $18(6,2 \%)$ & $58(20 \%)$ & $1(0,3 \%)$ \\
\hline 3.0 conteúdo é muito difícil de entender & $36(12,7 \%)$ & $138(47,6 \%)$ & $17(5,9 \%)$ & $95(32,8 \%)$ & $4(1,4 \%)$ \\
\hline 4.Demoro muito para ler (as instruções) & $22(7,6 \%)$ & $143(49,3 \%)$ & $53(18,3 \%)$ & $71(24,5 \%)$ & $1(0,3 \%)$ \\
\hline \multirow[t]{2}{*}{ 5. Eu preciso que alguém me ajude a ler } & $24(8,3 \%)$ & $115(39,7 \%)$ & $24(8,3 \%)$ & $123(42,4 \%)$ & $4(1,4 \%)$ \\
\hline & $4(1,4 \%)$ & $44(15,2 \%)$ & $12(4,1 \%)$ & $208(71,7 \%)$ & $22(7,6 \%)$ \\
\hline \multicolumn{6}{|l|}{ LITERACIA COMUNICATIVA } \\
\hline 6.Eu procuro informações em vários lugares & $3(1 \%)$ & $86(29,7 \%)$ & $10(3,4 \%)$ & $169(58,3 \%)$ & $22(7,6 \%)$ \\
\hline \multicolumn{6}{|l|}{ 7.Eu encontro a informação que preciso } \\
\hline \multicolumn{6}{|l|}{ 8. Eu entendo a informação encontrada } \\
\hline $\begin{array}{l}\text { 9.Eu falo minha opinião sobre a doença ao meu } \\
\text { médico, familiares ou amigos } \\
\text { 10.Eu coloco em prática as informações }\end{array}$ & $1(0,3 \%)$ & $33(11,4 \%)$ & $12(4,1 \%)$ & $215(74,1 \%)$ & $29(10 \%)$ \\
\hline encontradas no meu dia a dia & $2(0,7 \%)$ & $16(5,5 \%)$ & $15(5,2 \%)$ & $224(77,2 \%)$ & $33(11,4 \%)$ \\
\hline LITERACIA CRÍTICA & & & & & \\
\hline $\begin{array}{l}\text { 11.Eu sei quando as informações são boas no meu } \\
\text { caso }\end{array}$ & $1(0,3 \%)$ & $23(7,9 \%)$ & $19(6,6 \%)$ & $235(81 \%)$ & $12(4,1 \%)$ \\
\hline $\begin{array}{l}\text { 12.Eu levo em conta se as informações são } \\
\text { verdadeiras }\end{array}$ & $1(0,3 \%)$ & $40(13,8 \%)$ & $30(10,3 \%)$ & $196(67,6 \%)$ & $23(7,9 \%)$ \\
\hline $\begin{array}{l}\text { 13.Eu tenho conhecimento para julgar se as } \\
\text { informações são confiáveis }\end{array}$ & $5(1,7 \%)$ & $116(40 \%)$ & $36(12,4 \%)$ & $118(40,7 \%)$ & $15(5,2 \%)$ \\
\hline $\begin{array}{l}\text { 14.Eu pego informações que me ajudam a tomar } \\
\text { decisões de como melhorar minha saúde }\end{array}$ & $1(1 \%)$ & $13(4,5 \%)$ & $11(3,8 \%)$ & $232(80,3 \%)$ & $30(10,3 \%)$ \\
\hline
\end{tabular}

\footnotetext{
* Questionário HLS-14 (Suka et al, 2013) e (Batista et al, 2020). Fonte: Autores.
}

$\mathrm{Na}$ análise bivariada entre as variáveis independentes e as dimensões de literacia, houve associação significativa com 


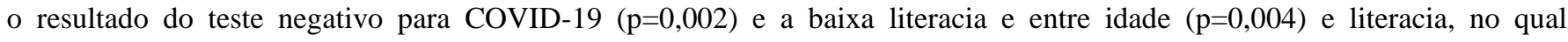
participantes de menor idade (18 a 39 anos) apresentaram alta literacia. Quanto aos hábitos de saúde durante a pandemia, houve associação entre o não conhecimento sobre distanciamento social como medida preventiva e baixa literacia $(\mathrm{p}=0,047)$ (Tabela 3).

Tabela 3. Distribuição de variáveis bivariadas para literacia alta e baixa em saúde.

\begin{tabular}{|c|c|c|c|c|}
\hline \multirow{2}{*}{\multicolumn{2}{|c|}{ Variáveis }} & \multirow{2}{*}{$\begin{array}{c}\text { Baixa LS } \\
\text { n }(\%)\end{array}$} & \multirow{2}{*}{$\begin{array}{c}\text { Alta LS } \\
\text { n (\%) }\end{array}$} & \multirow{2}{*}{ p-valor } \\
\hline & & & & \\
\hline \multirow{2}{*}{$\begin{array}{c}\text { Resultado do teste para } \\
\text { COVID-19 }\end{array}$} & Negativos & $81(54,4 \%)$ & $68(45,6 \%)$ & \multirow{2}{*}{0,002} \\
\hline & Positivos & $51(36,2 \%)$ & $90(63,8 \%)$ & \\
\hline \multirow{3}{*}{ Idade (em anos) } & 18 a 19 & $52(38,5 \%)$ & $83(61,5 \%)$ & \multirow{3}{*}{0,004} \\
\hline & 40 a 59 & $51(45,5 \%)$ & $61(54,5 \%)$ & \\
\hline & 50 ou mais & $29(67,4 \%)$ & $14(32,6 \%)$ & \\
\hline \multirow{2}{*}{ Sexo } & Feminino & $93(48,9 \%)$ & $89(51,1 \%)$ & \multirow{2}{*}{0.341} \\
\hline & Masculino & $48(45,3 \%)$ & $58(54,6 \%)$ & \\
\hline \multirow{3}{*}{ Escolaridade } & Ensino fundamental & $33(75 \%)$ & $11(25 \%)$ & \multirow{3}{*}{0,000} \\
\hline & Ensino médio & $58(51,3 \%)$ & $55(48,7 \%)$ & \\
\hline & Ensino superior & $47(37 \%)$ & $80(63 \%)$ & \\
\hline \multirow{3}{*}{ Renda pessoal } & Sem renda ou até 1 salário* & $5(62,5 \%)$ & $3(37,5 \%)$ & \multirow{3}{*}{0,080} \\
\hline & 1 a 3 salários & $59(51,8 \%)$ & $55(48,2 \%)$ & \\
\hline & 3 ou mais salários & $56(39,2 \%)$ & $87(60,8 \%)$ & \\
\hline \multirow{4}{*}{$\begin{array}{l}\text { Interferência do } \\
\text { COVID-19 na rotina }\end{array}$} & Não sai ou sai para mercado, farmácia e urgência & $83(46,1 \%)$ & $97(53,9 \%)$ & \multirow{4}{*}{0,965} \\
\hline & Sai para trabalhar & & & \\
\hline & Não alterou a rotina & $44(44,4 \%)$ & $55(55,6 \%)$ & \\
\hline & & $5(45,5 \%)$ & $6(54,5 \%)$ & \\
\hline \multirow{2}{*}{ Orientações preventivas } & Seguiu as orientações de prevenção e família & $109(45 \%)$ & $133(55 \%)$ & \multirow{2}{*}{0,715} \\
\hline & Não seguiu as orientações de prevenção & $23(47,9 \%)$ & $25(52,1 \%)$ & \\
\hline \multirow{2}{*}{ Isolamento } & Seguiu & $90(45,5 \%)$ & $108(54,5 \%)$ & \multirow{2}{*}{0,975} \\
\hline & Não seguiu & $42(45,7 \%)$ & $50(54,3 \%)$ & \\
\hline \multirow{2}{*}{$\begin{array}{l}\text { Evitar contato com } \\
\text { alguém próximo } \\
\text { infectado }\end{array}$} & Não evitar contato & $110(48,2 \%)$ & $118(51,8 \%)$ & \multirow[b]{2}{*}{0,074} \\
\hline & Evitar contato & $22(35,5 \%)$ & $40(64,5 \%)$ & \\
\hline \multirow{2}{*}{$\begin{array}{c}\text { Conhecimento } \\
\text { preventivo do } \\
\text { distanciamento social }\end{array}$} & Não manter distanciamento & $62(52,5 \%)$ & $56(47,7 \%)$ & \multirow{2}{*}{0,047} \\
\hline & Manter distanciamento & $70(40,7 \%)$ & $102(59,3 \%)$ & \\
\hline \multirow{2}{*}{ Uso de máscara } & Não fez uso de máscaras & $45(48,9 \%)$ & $47(51,1 \%)$ & \multirow{2}{*}{0,429} \\
\hline & Fez uso de máscaras & $87(43,9 \%)$ & $111(56,1 \%)$ & \\
\hline Autopercepção: Já & Não & $26(46,4 \%)$ & $30(53,6 \%)$ & \multirow{6}{*}{0,631} \\
\hline existe tratamento para & Sim & $95(44,4 \%)$ & $119(55,6 \%)$ & \\
\hline COVID-19 & Não sei & $11(55 \%) /$ & $9(45 \%)$ & \\
\hline \multirow{3}{*}{$\begin{array}{c}\text { Autopercepção: já } \\
\text { existe vacina para a } \\
\text { COVID-19 }\end{array}$} & Sim & $13(38,2 \%)$ & $21(61,8 \%)$ & \\
\hline & Não & $110(46,2 \%)$ & $128(53,8 \%)$ & \\
\hline & Não sei & $9(50 \%)$ & $9(50 \%)$ & \\
\hline & Pouco & $18(64,3 \%)$ & $10(35,7 \%)$ & \\
\hline Conhecimento & Médio & $84(41,8 \%)$ & $117(58,2 \%)$ & 0,066 \\
\hline & Muito & $30(49,2 \%)$ & $31(50,8 \%)$ & \\
\hline
\end{tabular}

Fonte: Autores.

Também foi realizou-se a análise de mediação para verificar o efeito da literacia em saúde no resultado do teste rápido para COVID-19, entretanto, não verificou-se efeito estatisticamente significante para os resultados positivos, sendo o coeficiente de beta 0,023 (IC95\%=0,024; -0,07). Em relação ao valor de p=0,339 e idade houve significância no efeito sobre os resultados, mediando à associação entre literacia e soropositividade. No modelo idade houve coeficiente $-0,04$ (IC95\%= $0,06 ;-0,02)$ e $\mathrm{p}<0,001$. 


\section{Discussão}

O presente estudo é pioneiro em território brasileiro na associação da adesão às medidas de prevenção contra a COVID-19 com a literacia em saúde dos participantes. No presente estudo houve associação entre a baixa literacia e a falta de conhecimento sobre a importância do distanciamento social como medida de prevenção. Além dessa variável, a baixa literacia foi associada com menor escolaridade e maior idade. O acesso a essas informações para a promoção de saúde é de extrema importância, uma vez que a literacia em saúde pode ser um determinante da saúde mediador de melhores desfechos em saúde (Nutbeam, 2000).

A partir deste estudo evidenciou-se que a maior parte da amostra apresentou alta literacia. Este resultado não foi encontrado em outro estudo até o momento, porém em pesquisa nacional recente, Oscalices et al. (2019) evidenciou que uma maior porcentagem de brasileiros apresentou baixa literacia.

No que se refere à escolaridade, o grupo que concluiu o ensino superior teve uma maior prevalência de indivíduos com alta literacia. Por outro lado, bem mais da metade daqueles que concluíram apenas o ensino fundamental foi avaliado com baixa literacia. Como encontrado em outros estudos a escolaridade está diretamente relacionado à literacia em saúde, uma vez que a educação dá acesso à ferramentas de busca e conhecimento necessário para que os indivíduos saibam administrar melhor sua saúde e sua qualidade de vida (Cavaco \& Santos, 2012). É importante ressaltar que o Índice de Desenvolvimento Humano Municipal (IDHM) do município de Jundiaí, considerado alto (IDHM=0,822) (IBGE, 2010), pode ter refletido diretamente nestas variáveis sociodemográficas, cuja distribuição, entretanto, apresentou-se em concordância com a literacia média apresentada pelo grupo estudado (Programa das Nações Unidas para o Desenvolvimento, n.d.).

Outro ponto relevante também a ser considerado, refere-se à maioria dos participantes possuir idade menor que 60 anos. Como já demonstrado por Baker et al. (2000), Berens et al. (2016) e Sørensen et al. (2015) a baixa literacia em saúde é associada a maior idade. Dessa forma, pode-se que os mais idosos tendem a ter maiores dificuldades de compreensão quanto a informações relacionadas à saúde.

Neste contexto de pandemia, muitas informações e falsas informações em saúde foram disseminadas por veículos de informação, sendo que o distanciamento foi de fato comprovadamente uma das medidas mais importantes para a prevenção da doença e a conter a propagação da doença. Contudo no estudo a baixa literacia foi associada com a falta de conhecimento do distanciamento social como forma de prevenção para a COVID-19. Logo, pode-se perceber que o conhecimento acerca das maneiras de se evitar o contágio de doenças infecciosas são menos familiares àqueles que apresentam baixa literacia, como já havia proposto Castro-sánchez et al. (2016). De acordo com estudo de Apolinário et al. (2014), muitos indivíduos não aderem a comportamentos de saúde preventivos por não compreenderem de forma integral as informações em saúde disponíveis. Por este motivo, identificar na população quais são as dificuldades encontradas para a adoção dessas práticas é essencial para o sucesso de políticas de promoção da saúde. Dessa forma, pode-se inferir que a falta de conhecimento em relação à saúde pode estar associada a uma menor taxa de adesão a medidas de precaução no que tange a doenças infecciosas (Oscalices, et al., 2019).

Tal associação pode ser explicada pela falta de incentivo do governo Federal no que tange à adoção de medidas preventivas e distanciamento social. Até mesmo os participantes com alta literacia não mantiveram o distanciamento adequadamente, já que as autoridades não foram capazes de instruir a população quanto aos perigos das aglomerações (Ferigato et al., 2020). Apesar de o cenário descrito ter sido predominante no Brasil como um todo, é importante ressaltar que o município de Jundiaí teve atitudes para combater a pandemia. Dentre uma das ações adotadas foi à distribuição de máscaras de proteção pela prefeitura nos terminais de ônibus da cidade e campanhas recomendando o distanciamento social (Prefeitura de Jundiaí, n.d.). 
Em relação aos comportamentos preventivos, mais da metade dos indivíduos da amostra relatou mudanças em seus hábitos diários e, principalmente, adesão às medidas de isolamento social. Esses dados se aproximam dos valores fornecidos pelo Sistema de Monitoramento Inteligente do Governo de São Paulo, o qual utiliza informações fornecidas por operadoras de telefonia móvel para analisar a tendência de deslocamento e apontar a eficácia das medidas de distanciamento social. No período estudado, apesar das variações, o índice de isolamento foi mediano (SEADE [Recuperado em 22, novembro, 2021]), Pode-se especular que isso tenha contribuído para o aumento das contaminações tanto em indivíduos com alta literacia quando em indivíduos com baixa literacia.

A respeito da compreensão de literacia funcional foram feitas perguntas sobre a leitura das bulas de medicamentos, como "Eu encontro palavras que não consigo ler" com concordância em mais da metade dos participantes, além das perguntas de número dois, três e quatro, demonstrando que muitos tinham dificuldade de interpretação e leitura de palavras do contexto da saúde. Este resultado reitera como é complexo para os indivíduos compreender as instruções de prescrição, dosagem de medicamentos e possíveis riscos de saúde (Nutbeam, 2000). Tal fato torna-se alarmante uma vez que demonstra que a dificuldade das pessoas em administrar sua própria saúde, pode contribuir para uma diminuição da qualidade de vida, indicando um ponto vulnerável a ser melhorado. A LS é reconhecida pela Organização Mundial da Saúde (OMS) como pilar da promoção da saúde e importante marcador de desigualdade em saúde, pode auxiliar os profissionais de saúde no atendimento a essa população (Loureiro et al., 2015). No Brasil, o aspecto da literacia funcional com relação à compreensão de recomendações em saúde, é essencial na pandemia da COVID-19, pois medicamentos como a cloroquina e hidroxicloroquina, que obtiveram um aumento de 68\% das vendas durante o período (Conselho Federal de Farmácia, n.d.), foram divulgados como tratamento precoce e prevenção da infecção causada pelo SARS-COV-2, sendo que estas são medicações comprovadamente sem eficácia para esta condição (Galeris et al., 2020). A promoção da literacia em saúde poderia favorecer tomada de decisões em saúde em concordância com evidências científicas.

Quando questionados "Eu procuro informações sobre a COVID-19 em vários lugares", a maioria da amostra do presente estudo relatou discordar da frase, o que evidencia uma deficiência na busca ativa de informações sobre a doença. Considerando que a internet é uma das principais fontes de conteúdo e ao mesmo tempo repleta de informações falsas em saúde, este pode ser um fator de impacto sobre o enfrentamento da pandemia. Isso pode ser explicado pelo fato da população ter a tendência de não procurar os assuntos de forma mais aprofundada em sites com embasamento científico e se apegar a informações não confiáveis em mídias sociais, de modo que as medidas de enfrentamento à pandemia que são relevantes são menosprezadas e desconhecidas, como encontrado no presente estudo (Garcia e Duarte, 2020).

Na pergunta "Eu sei quando as informações sobre a COVID-19 são boas no meu caso", foi utilizada para medir, de maneira combinada, a literacia crítica e comunicativa (Suka, et al., 2013). Conforme obtido, quase a totalidade da amostra discorda dessa afirmação, indicando uma menor percepção da capacidade de ponderação sobre a plausibilidade da aplicação de informações em saúde sobre o coronavírus no dia a dia dos entrevistados. Tal fato pode ser explicado pela influência do fenômeno denominado "Infodemia", presente durante a pandemia pelo novo Coronavírus, no qual há o acesso a informações diversas e disseminadas com tanta facilidade por meio das redes sociais, que resultam na dificuldade de o indivíduo distinguir aquilo que é pertinente (Garcia e Duarte, 2020). Além disso, o governo brasileiro não estabeleceu um planejamento concreto para enfrentar a pandemia nem para transmitir informação de confiança para a população.

No presente estudo, indivíduos que apresentaram resultado positivo para IgG e IgM para COVID-19 apresentaram maior prevalência de alta literacia, contrariamente ao esperado. Porém, após análise de mediação verifica-se que a idade foi mediadora deste resultado, sendo que os mais jovens foram os que apresentaram maior prevalência de resultados positivos (resultados não apresentados) e também maior prevalência de alta literacia. Assim, a idade e outros fatores não apresentados no presente estudo podem trazer uma confusão com relação ao resultado do teste e a associação com literacia. 
Ressalta-se que a literacia em saúde está mais associada à comportamentos em saúde e conhecimento, sendo identificada como um fator intermediário da saúde. Estudos têm demonstrado resultados inconclusivos com relação aos desfechos clínicos, devido a grande relevância dos determinantes sociais da saúde nos aspectos clínicos (Tenani et al., 2021). Entretanto, estudos futuros devem continuar investigando o papel da literacia em saúde no contexto de um modelo teórico conceitual dos determinantes da saúde.

Estudo que avaliou os gradientes socioeconômicos de saúde e COVID-19 observou que a população foi acometida pela doença em todos os níveis desses gradientes e recomenda assim que haja investimento na literacia em saúde da população como uma intervenção relevante para o enfrentamento de pandemias, melhorando a capacidade de buscar informações fidedignas e avaliar essas informações para a tomada de decisão em saúde. Ressalta-se que o foco na promoção de saúde neste contexto não deve ser exclusividade das pessoas doentes, mas sim uma realidade de toda a população, para que possa haver prevençao e difusão de práticas de higiene eficazes entre todos, controlando o cenário pandêmico (Chung et al., 2020).

$\mathrm{O}$ estudo tem uma limitação do delineamento transversal que não traz em si a avaliação de risco e causalidade, e também a coleta de dados por telefone pode trazer em si um viés de informação, tendo em vista a dificuldade de disponilidade dos participantes para responder o questionário proposto. Porém, ressalta-se como forte potencial o delineamento amostral representativo da população, e o sorteio aleatório dos participantes tendo robustez no processo amostral.

Com isso, os serviços de saúde fazendo uso da literacia em saúde, podem direcionar medidas públicas e implantar o uso de ferramentas de comunicação efetivas para transmissão do conhecimento para esses indivíduos.

\section{Conclusão}

Houve associação entre a baixa literacia, a falta de conhecimento sobre a capacidade preventiva do distanciamento social, a idade dos participantes e escolaridade. Os resultados deste estudo demonstram a importância do investimento em políticas públicas adequadas e individualizadas, tendo como foco a literacia em saúde, para o fornecimento e acesso a informações que permitam a conscientização e informatização desse público, para que possam tomar as melhores decisões em saúde e controlar o impacto da pandemia. Estudos futuros devem aprofundar no conhecimento do papel da LS na infecção pela COVID-19 e adoção de medidas preventivas considerando os efeitos confundidores da idade.

\section{Referências}

Abdel-Latif M. (2020). The enigma of health literacy and COVID-19 pandemic. Public health, 185, 95-96.

Apolinario, D., Mansur, L. L., Carthery-Goulart, M. T., Brucki, S. M., \& Nitrini, R. (2014). Detecting limited health literacy in Brazil: development of a multidimensional screening tool. Health promotion international, 29(1), 5-14.

Badr, H. S., Du, H., Marshall, M., Dong, E., Squire, M. M., \& Gardner, L. M. (2020). Association between mobility patterns and COVID-19 transmission in the USA: a mathematical modelling study. The Lancet. Infectious diseases, 20(11), 1247-1254.

Baker, D. W., Gazmararian, J. A., Sudano, J., \& Patterson, M. (2000). The association between age and health literacy among elderly persons. The journals of gerontology. Series B, Psychological sciences and social sciences, 55(6), S368-S374.

Batista, M. J., Marques, A., Silva Junior, M. F., Alencar, G. P., \& Sousa, M. (2020). Translation, cross-cultural adaptation and psychometric evaluation of Brazilian Portuguese version of the 14-item Health Literacy Scale. Tradução, adaptação transcultural e avaliação psicométrica da versão em português (brasileiro) do 14-item Health Literacy Scale. Ciência \& Saúde coletiva, 25(7), 2847-2857.

Berens, E. M., Vogt, D., Messer, M., Hurrelmann, K., \& Schaeffer, D. (2016). Health literacy among different age groups in Germany: results of a crosssectional survey. BMC public health, 16(1), 1151.

Castro-Sánchez, E., Chang, P., Vila-Candel, R., Escobedo, A. A., \& Holmes, A. H. (2016). Health literacy and infectious diseases: why does it matter? International journal of infectious diseases: IJID, 43, 103-110.

Cavaco, A., \& Santos, A. L. (2012). Avaliação da legibilidade de folhetos informativos e literacia em saúde. Revista de Saúde Pública, 46, 918-922.

Chung, R. Y., Dong, D., \& Li, M. M. (2020). Socioeconomic gradient in health and the covid-19 outbreak. BMJ (Clinical research ed.), 369 , m1329. 
Conselho Federal de Farmácia. (n.d.). Levantamento mostra como o medo da Covid-19 impactou venda de medicamentos. https://www.cff.org.br/noticia.php?id=5747.

Ferigato, S., Fernandez, M., Amorim, M., Ambrogi, I., Fernandes, L., \& Pacheco, R. (2020). The Brazilian Government's mistakes in responding to the COVID-19 pandemic. Lancet (London, England), 396(10263), 1636.

Garcia, L. P., \& Duarte, E. (2020). Infodemia: excesso de quantidade em detrimento da qualidade das informações sobre a COVID-19. Epidemiol. Serv. Saúde, 29(4).

Geleris, J., Sun, Y., Platt, J., Zucker, J., Baldwin, M., Hripcsak, G., Labella, A., Manson, D. K., Kubin, C., Barr, R. G., Sobieszczyk, M. E., \& Schluger, N. W. (2020). Observational Study of Hydroxychloroquine in Hospitalized Patients with Covid-19. The New England journal of medicine, 382 (25), $2411-2418$.

Governo do Estado de São Paulo. (2020). Decreto n. 64.881, de 22 de março de 2020. Decreta quarentena no Estado de São Paulo, no contexto da pandemia do Covid-19 (Novo Coronavírus), e dá providencias complementares. https://www.saopaulo.sp.gov.br/wp-content/uploads/2020/03/decreto-quarentena.pdf.

Instituto Brasileiro de Geografia e Estatística. (2010). Censo demográfico 2010. https://www.ibge.gov.br/estatisticas/sociais/saude/9662-censo-demografico2010.html?edicao=9758\&t=resultados.

Košir, U., \& Sørensen, K. (2020). COVID-19: the key to flattening the curve is health literacy. Perspectives in public health, 1757913920936717. Advance online publication.

Loureiro, I. (2015). A literacia em saúde, as políticas e a participação do cidadão. Revista portuguesa de saúde pública, 33(1), 1-1.

Ministério da Saúde. (2019). Doença pelo coronavirus $2019 \quad$ (covid-19) Boletim https://portalarquivos.saude.gov.br/images/pdf/2020/April/18/2020-04-17---BE11---Boletim-do-COE-21h.pdf.

Nutbeam, D. (2000). Health literacy as a public health goal: a challenge for contemporary health education and communication strategies into the 21 st century. Health promotion international, 15(3), 259-26.

Okan, O., Bollweg, T. M., Berens, E. M., Hurrelmann, K., Bauer, U., \& Schaeffer, D. (2020). Coronavirus-Related Health Literacy: A Cross-Sectional Study in Adults during the COVID-19 Infodemic in Germany. International journal of environmental research and public health, 17(15), 5503.

Oscalices, M. I. L., Okuno, M. F. P., Lopes, M. C. B. T., Batista, R. E. A., \& Campanharo, C. R. V. (2019). Literacia em saúde e adesão ao tratamento de pacientes com insuficiência cardíaca. Revista da Escola de Enfermagem da USP, 53.

Prefeitura de Jundiaí. (n.d.). Jundiaí distribui 20 mil máscaras doadas nos terminais urbanos. https://jundiai.sp.gov.br/noticias/2020/04/19/jundiai-distribui20-mil-mascaras-doadas-nos-terminais-urbanos/

Programa das Nações Unidas para o Desenvolvimento. (n.d.). $O$ que é Índice de Desenvolvimento Humano. https://www.br.undp.org/content/brazil/pt/home/idh0/conceitos/o-que-e-o-idhm.html.

Shen, M., Peng, Z., Xiao, Y., \& Zhang, L. (2020). Modeling the Epidemic Trend of the 2019 Novel Coronavirus Outbreak in China. Innovation (New York, N.Y.), 1(3), 100048 .

Sørensen, K., Pelikan, J. M., Röthlin, F., Ganahl, K., Slonska, Z., Doyle, G., Fullam, J., Kondilis, B., Agrafiotis, D., Uiters, E., Falcon, M., Mensing, M., Tchamov, K., van den Broucke, S., Brand, H., \& HLS-EU Consortium (2015). Health literacy in Europe: comparative results of the European health literacy survey (HLS-EU). European journal of public health, 25(6), 1053-1058.

Suka, M., Odajima, T., Kasai, M., Igarashi, A., Ishikawa, H., Kusama, M., Nakayama, T., Sumitani, M., \& Sugimori, H. (2013). The 14-item health literacy scale for Japanese adults (HLS-14). Environmental health and preventive medicine, 18(5), 407-415.

Tenani, C.F., Silva Junior M.F., Sousa M.L.R., Batista M.J. O papel da literacia em saúde como fator associado às perdas dentárias. (2021). Revista de Saúde Pública. In press.

Wellenius, G. A., Vispute, S., Espinosa, V., Fabrikant, A., Tsai, T. C., Hennessy, J., ... \& Gabrilovich, E. (2021). Impacts of social distancing policies on mobility and COVID-19 case growth in the US. Nature communications, 12(1), 1-7.

World Health Organization. (2019). Coronavirus disease (COVID-19) advice for the public. https://www.who.int/emergencies/diseases/novel-coronavirus2019/advice-for-public.

World Health Organization. (2020). General's opening remarks at the media briefing on COVID-19. https://www.who.int/dg/speeches/detail/who-directorgeneral-s-opening-remarks-at-the-media-briefing-on-covid-19---11-march-2020.

Yesudhas, D., Srivastava, A., \& Gromiha, M. M. (2021). COVID-19 outbreak: history, mechanism, transmission, structural studies and therapeutics. Infection, 49(2), 199-213.

Zhang, Z., Yao, W., Wang, Y., Long, C., \& Fu, X. (2020). Wuhan and Hubei COVID-19 mortality analysis reveals the critical role of timely supply of medical resources. The Journal of infection, 81(1), 147-178. 